Colloque international « Paulo Emilio Salles Gomes et Jean Vigo : cinéphilie, littérature et patrimoine cinématographique »

Montpellier-Perpignan, 28-30 mai 2013

\title{
Lilia Oliveira
}

\section{OpenEdition}

\section{Journals}

Édition électronique

URL : https://journals.openedition.org/1895/4690

DOI : $10.4000 / 1895.4690$

ISSN : $1960-6176$

Éditeur

Association française de recherche sur l'histoire du cinéma (AFRHC)

Édition imprimée

Date de publication : 1 juin 2013

Pagination : 180-185

ISBN : 978-2-37029-070-0

ISSN : 0769-0959

\section{Référence électronique}

Lilia Oliveira, «Colloque international « Paulo Emilio Salles Gomes et Jean Vigo : cinéphilie, littérature et patrimoine cinématographique » », 1895. Mille huit cent quatre-vingt-quinze [En ligne], 70 | 2013, mis en ligne le 01 juin 2016, consulté le 16 avril 2022. URL : http://journals.openedition.org/1895/4690 DOI : https://doi.org/10.4000/1895.4690 
Colloque international

«Paulo Emilio Salles Gomes et Jean Vigo : cinéphilie, littérature et patrimoine cinématographique» (Montpellier-Perpignan, 28-30 mai 2013)

Les 28, 29 ET 30 MAI 2013, s'est tenu à l'Université Paul Valéry - Montpellier 3 et à l'Institut Jean Vigo à Perpignan, un colloque international organisé conjointement par le Centre de Recherche RIRRA 21, par l'Institut Jean Vigo et par la Cinémathèque brésilienne de São Paulo. L'objectif était de mettre en évidence les échanges culturels France-Brésil aussi bien que de décrire l'univers de la cinéphilie francobrésilienne dans une perspective socio-historique autour de l'œuvre de Jean Vigo et de son premier biographe, le critique brésilien Paulo Emilio Salles Gomes.

Michel Cadé, président de la Cinémathèque Eurorégionale Institut Jean Vigo, Marie-Ève Therenty, directrice du Centre du Recherche RIRRA 21, Adilson Mendes, chercheur associé à la Cinemateca brasileira et Sebastiana Gomes Lisboa, chercheuse indépendante associée au groupe de recherche RIRRA 21, ont ouvert la discussion qui rassemblait de nombreux chercheurs de différentes domaines cinéma, littérature, philosophie et histoire -, venus du Brésil, de Suisse, de France, de Grande-Bretagne et des États-Unis, afin de mettre en lumière les liens entre l'œuvre de Paulo Emilio (comme on dit au Brésil) et celle de Jean Vigo. C'est d'ailleurs sous le regard et l'écoute attentive de Luce Vigo, sa fille, elle aussi biographe tardive de son père, que tout le colloque se déroula.

La première communication était due à Pierre Lherminier, historien du cinéma, également biographe de Jean Vigo, dont on fit la lecture en raison de son empêchement à se déplacer; elle témoigna de l'importance inaugurale du travail de Salles Gomes comme du rôle que joua la redécouverte de Vigo dans la culture cinéphilique française.

\section{Paulo Emilio Salles Gomes, le critique, le biographe, l'écrivain}

Quels liens établir entre Salles Gomes et Vigo, le Brésil et la France, pourquoi ce colloque conjoignant deux figures et des pays si lointains et différents? La première intervention d'Adilson Mendes, dont la thèse de doctorat était consacrée à la trajectoire intellectuelle de Paulo Emilio, a servi à la fois de "présentation du personnage brésilien" au public français présent et de compréhension des liens entre les deux auteurs. En proposant de décortiquer le "projet intellectuel de Paulo Emilio", en soulignant les différentes facettes du Brésilien ( cinéphile, critique, militant, historien, écrivain), distinctes et, en même temps, complémentaires, Mendes a choisi de se centrer sur son travail critique. Il a tout d'abord mis en évidence l'influence de Paul Valéry dans la prose littéraire de Paulo Emilio - ses échos pouvant être perçus dans son Jean Vigo -, tout en soulignant que c'était justement cette prose littéraire qui amalgamait le souvenir, la fantaisie et l'érudition si importants et présents dans le projet intellectuel du Brésilien. En même temps, il a relevé l'hétérodoxie théorique de Paulo Emilio, reflet direct d'un esprit libre et d'un incroyable appétit intellectuel, développé davantage lors de ses séjours à Paris où il fréquenta le Cercle du cinéma, rencontrant Henri Langlois et Georges Franju, découvrant Octobre, entre autres films d'Eisenstein, ainsi que la littérature née du débat autour du parlant. C'est grâce à ce premier séjour parisien que Paulo Emilio a pu inaugurer au Brésil, au début des années 1940, le genre de «l'essai cinématographique» avec la création de la revue Clima. Cette phase de la vie du critique brésilien fut illustrée par sa "surprenante» analyse de Citizen Kane, où, avant quiconque, il mit l'accent sur le rôle de la profondeur de champ. La découverte du "Rimbaud du cinéma», Jean Vigo, et de ses films poétiquement révolutionnaires date du deuxième séjour à Paris (1946-1954). Alliant son intuition critique, son travail d'historien, sa connaissance matérielle de conservateur de films et son esprit d'essayiste, le critique brésilien écrivit la première 
biographie du cinéaste. Son Jean Vigo, fut publié en 1957 aux éditions du Seuil dont Chris Marker conseillait la collection cinéma. Dans un deuxième temps, Mendes mit en lumière son rôle dans la «naissance» et le développement du Cinema Novo, notamment de Glauber Rocha. Pourtant Paulo Emilio n'a jamais écrit sur aucun film de Rocha et très peu sur les films du Cinema Novo. Pour Mendes le "projet intellectuel de Paulo Emilio" était de penser une culture et ses contradictions historiques à partir du phénomène cinématographique, en utilisant différents types de savoirs, liés les uns aux autres, par une prose littéraire capable de rendre transmissible la discipline de l'histoire du cinéma. Parmi les différentes facettes de Paulo Emilio, il y a donc la littéraire. Son livre de fiction de 1977, As três mulheres de três PPP's, ( P... comme Polydore, Actes Sud, 1986), en témoigne. Composé de trois nouvelles différentes autour d'un même narrateur, Polydore (PPP), le livre raconte des histoires de couples de la bourgeoisie paulistana (de São Paulo), tout en faisant une analyse critique de cette société. José Antonio Pasta Jr., professeur de littérature brésilienne à l'Université de São Paulo, a souligné combien, grâce à son style original, le renouvellement du sens des mots et son esprit critique très aigu, le livre de Paulo Emilio avait fait date pour la critique, et fut même crédité d'ouvrir un courant historique et esthétique dépassant le modernisme. On y vit «la meilleure prose brésilienne depuis Guimarães Rosa ". Pasta compara les formes - l'humour, le sarcasme, l'ironie - et les sujets de P... comme Polydore, à ceux de Machado de Assis, autre icône de la littérature brésilienne, très connu pour ses critiques sociales mordantes et actuelles. Avec cette différence que le livre de Paulo Emilio visait la bassesse d'une classe dominante en pleine décomposition dans les années 1970. Cette façon de mélanger les différents registres (haut et bas), selon Pasta, engendrait une forme littéraire hybride entre le grand art et la littérature érotique populaire, cette dernière transposée au cinéma par le genre pornochanchada, très mal vu à l'époque, mais ayant pourtant, pour Paulo Emilio, une fonction dénonciatrice.

\section{Paulo Emilio Salles Gomes, l'homme de gauche}

Cette fonction dénonciatrice fut l'une des ses préoccupations majeures en tant que citoyen et publiciste. Né dans une famille aisée avec un père secrétaire d'État et homme d'affaires, il s'est très tôt senti mal à l'aise dans sa condition de bourgeois. Se sentant concerné par les questions de justice et d'égalité sociales, il s'engagea très jeune dans le communisme. François Albera rappela cet aspect de l'engagement de Salles Gomes, incarcéré en 1935 après l'échec de l'Intentona Comunista, l'insurrection contre le gouvernement de Getúlio Vargas lancée par le Parti communiste brésilien. Alors qu'on ne sait pas grand-chose de son rapport au cinéma jusque-là, $a$ fortiori s'il avait vu des films accordés à ses convictions révolutionnaires, c'est en s'évadant, en 1937, et en s'exilant en France que Salles Gomes découvrit le cinéma soviétique et le cinéma en général. Un rôle par conséquent fondateur: avec les films d'Eisenstein, Vertov, Poudovkine, etc., il découvre un cinéma qui se donne des objectifs sociaux et politiques et qui renouvelle les formes. C'est pourtant assez tardivement qu'il s'exprime sur ces films, à un moment où il a pris ses distances avec le militantisme et avec l'adhésion au communisme, après être revenu en France, y avoir fréquenté les ciné-clubs d'après-guerre et même l'Institut de filmologie. Il écrit alors dans le supplément littéraire du journal à grand tirage, O Estado de S. Paulo, et de 1957 à 1959, il commence une série d'articles sur Eisenstein suscités par la parution de la biographie de Marie Seton qui déclenche chez lui une réflexion sur les rapports de la vie privée et de l'engagement politique et lui font découvrir la dimension de penseur, de théoricien de l'auteur du Potemkine qui est près de l'emporter sur le cinéaste et qui, à ses yeux, domine le paysage: "Louis Delluc par le passé ou André Bazin actuellement font fréquemment preuve d'un grand talent, mais seuls quelques textes de Sergueï Mikhaïlovich Eisenstein donnent le sentiment d'une véritable grandeur intellectuelle", écrit-il. C'est l'occasion d'évoquer le contexte politique, la culture et le cinéma soviétiques (il a lu MacDonald, Victor Serge) mais aussi de faire cette 
déclaration de non-cinéphilie définissant son approche du cinéma: "Je constate une fois de plus combien l'appréciation des films est pauvre et peu stimulante lorsqu'elle se limite au champ cinématographique. Les virtualités et vertus des œuvres se fanent lorsqu'on les examine dans le compartiment étanche de la spécificité.» On peut donc penser que l'exemple du cinéma soviétique l'aida à réfléchir à l'édification d'un nouveau cinéma au Brésil, qui ne resterait plus prisonnier des modèles hollywoodiens. Tout le rapport au pathétique et au sacré qu'il retient chez Eisenstein ne se retrouve-t-il pas chez Glauber Rocha?

Isabelle Marinone revint sur le rapport à la politique en décrivant un Salles Gomes "anarchiste brésilien". Selon elle, percevant le politique à travers la poétique des œuvres filmiques s'il reconnut rapidement l'importance de l'avant-garde soviétique il accorda une place marquante au "réalisme poétique» et au cinéma du Front populaire comme propre à articuler un discours libertaire non dogmatique. On connaît son intérêt pour le passé d'Eugène Bonaventure de Vigo dit Miguel Almereyda, le père de Vigo, à qui, à l'origine, près d'un tiers de la biographie du cinéaste était consacré et dont l'éditeur français retrancha la plus grande partie en raison de son contenu politique. Dans ses articles de l'O Estado de S. Paulo (1956-1965), ainsi que dans la biographie de Vigo, on constate l'importance du travail de recherche, articulé avec des analyses esthétiques précises des films, et leur contextualisation socio-historique. Le Vigo appartiendrait à ce que Marinone appelle un "genre conversationnel», sensible et ciselé.

\section{Le Cinema Novo et le parricide}

Restant dans le champ de la «révolution», Mateus Araújo Silva mit en évidence l'importance de Paulo Emilio Salles Gomes pour le Cinema Novo - désireux de libération et porteur d'un message révolutionnaire -, tout en discutant son rôle, sa participation et son influence dans la formation et développement du mouvement cinématographique le plus important $\mathrm{du}$ Brésil. Pourquoi ce «silence paradoxal» du critique sur ce mouvement qu'il semblait appeler de ses vœux, qui se réclamait de lui? Il semblait naturel qu'il en devînt le porte-parole. "J'ai découvert le Cinema Novo chez moi!», aurait-il déclaré. Ce que traduit Araujo: "À l'intérieur de moi-même». Pensait-il que c'était aux jeunes de discuter du Cinema Novo, s'excluant de cette nouvelle étape révolutionnaire du pays? Ou ce refus d'écrire sur le Cinema Novo étaitil une forme de contestation envers la vision que promouvaient ses protagonistes? Araujo conclut que Paulo Emilio a été plus historien que critique au sujet du Cinema Novo, et qu'il avait écrit plus de critiques sur les films européens que sur des films brésiliens pendant sa trajectoire critique, même s'il était l'un de plus grands défenseurs du cinéma brésilien.

Sous un titre suggestif: "Le parricide, le cinéma d'auteur et le Vigo de Salles Gomes ", Lúcia Nagib, de l'Université de Reading, s'est focalisée sur la figure du parricide comme élément voilé mais central de l'approche biographique de Salles Gomes. Son point de départ a été une citation extraite du Jean Vigo: "Un après-midi du début d'août, [Jean Vigo] alla acheter des lacets pour les bottines d'Almereyda» (pp. 29-30). Or l'assassin utilisa ces mêmes lacets pour étrangler Almereyda dans sa cellule. Vigo aurait ainsi involontairement "tué» son père. Cette interprétation s'explique, selon Nagib, par la façon dont Salles Gomes a placé la phrase dans le texte, jetée accidentellement dans un paragraphe où elle ne se relie en rien avec ce qui vient avant ou après, comme s'il voulait offrir aux lecteurs les indices d'un crime à venir. Nagib a ainsi développé une analyse de cette biographie afin de montrer les contradictions inhérentes à son projet de combiner biographie, fiction et analyse des films. En même temps, elle a défendu l'idée que c'est grâce à ces contradictions que le dilemme au cœur même du cinéma d'auteur a pu être découvert, même s'il n'existait pas encore en tant que tel au moment où Salles Gomes terminait la biographie de Vigo, le besoin de tuer le "cinéma de papa» et toutes les figures paternelles étouffantes, en cherchant des substituts du père absent. Nagisa Oshima n'a-t-il pas déclaré: «La nonexistence de mon père a déterminé mon mode d'existence." 
Pour Luce Vigo, fille unique de Jean Vigo, critique de cinéma et, elle aussi, biographe de son père (Jean Vigo, une vie engagée dans le cinéma), il s'est agi "d'un rendez vous raté» avec son père, qui disparut quand elle n'avait que trois ans. Dans sa communication, elle a relevé l'importance du biographe pour les descendants du biographié. Dans son cas, elle a révélé que c'est à travers la biographie de Salles Gomes qu'elle a pu connaître vraiment son père, biographie qu'elle découvrit tardivement et seulement quand le livre parut, Salles Gomes lui ayant refusé de voir le manuscrit avant.

\section{Le genre de la biographie et la mythologie}

Luce Vigo avait souligné l'importance du travail de Lherminier, biographe de Vigo. Son livre, Jean Vigo, un cinéma singulier, paru en 1967, a fait l'objet de l'intervention de François Amy de la Bretèque, qui entreprit de le comparer à celui de Salles Gomes. D'abord au sein du genre de la biographie d'artiste, l'un de plus anciens genres littéraires de l'Occident. Selon lui la vie si singulière de Vigo aurait pu inciter à une approche plus légendaire, voire mythique du "personnage». Cependant les deux auteurs décidèrent de prendre, au contraire, une certaine distance. Puis de la Bretèque mit en parallèle les deux ouvrages, en analysant leurs couvertures, tables de matières et styles d'écriture, qu'il finit par qualifier de «quintessence de la biographie à la française», le travail de Salles Gomes servant de modèle à toute une production ultérieure, surtout celle de Lherminier qui, selon lui, en est consciemment l'héritier. Parmi ces héritiers, il y eut aussi Jacques Rozier qui, en 1964, réalisa un film de la série Cinéastes de notre temps, sous le titre «Jean Vigo, cinéaste de notre temps: essai de biographie filmée". Guillaume Boulangé a évoqué ce film composé de témoignages de plusieurs collaborateurs de Vigo, un film réalisé en toute liberté de style. Acteurs, producteurs, scénaristes et amis de Vigo parlent de lui trente ans après sa mort. On y découvre un Vigo très différent du "poète maudit» des histoires de cinéma, joyeux, plein d'humour, aimant la vie.

Maria Cecília Nogueira Coelho, professeure de philosophie à l'Université de Minas Gerais, a plongé, elle, dans l'univers de la mythologie grecque, en proposant une analyse de certains aspects de l'Atalante, ainsi que de la nouvelle Duas vezes com Helena [Deux fois avec Hélène], le premier des trois contes qui composent le livre Três Mulheres de PPPs [P... comme Polydor] de Paulo Emilio. Pour son étude de l'Atalante, Nogueira Coelho est partie des analyses de Paulo Emilio, dans son Jean Vigo, tout en se laissant influencer par ce qu'elle a nommé un "vice professionnel» de ceux qui étudient la culture grécoromaine, pour qui nomen est omen [le nom est un présage]. Le «vice» pouvant justifier son choix d'avoir comme objets d'étude, pour cette communication, le nom donné au bateau dans l'œuvre de Vigo, "L'Atalante», ainsi que les noms des personnages du conte de Paulo Emilio - Hélène et Polydore. Tous ayant des implications mythologiques.

Après avoir présenté brièvement le mythe d'Atalante (dans sa version arcadienne) - cette héroïne de la mythologie grecque abandonnée par son père sur une montagne (il ne voulait que des fils), nourrie par une ourse puis recueillie par des chasseurs, devint ellemême une chasseresse très agile et plus rapide que n'importe quel mortel - Nogueira Coelho nous a aussi rappelé son étymologie: celle qui a le même poids, l'égale (femme = homme). Sinon, la moins courante: l'intolérante, celle qui ne supporte pas. Atalante qui a des liens très forts avec le personnage de Juliette, une paysanne qui s'est décidée à épouser quelqu'un qui n'était pas de son village et qui l'a emmenée au loin sur un bateau. Seule femme parmi trois hommes, élément étranger qui va changer la routine du bateau au début et va rétablir son harmonie à la fin. Dans un deuxième temps, Nogueira Coelho a changé de mythe pour analyser cette fois-ci le conte de Paulo Emilio. Hélène, prénom courant au Brésil de l'époque, acquiert, selon elle, une nouvelle signification associé au nom Polydore, les deux noms faisant partie d'un même mythe grecque. Hélène est la célèbre spartiate à qui le prince Pâris rend visite tandis que Ménélas, son mari, était parti en voyage. Polydore est le frère cadet de Pâris. Or, chez Paulo Emilio, c'est Hélène l'épouse du professeur Alberto - qui, en l'absence de 
son mari, reçoit la visite du beau Polydore, en subvertissant ainsi la trame du mythe grecque. C'est elle qui séduit. Une histoire qui semble, selon Nogueira Coelho, avoir des rapports avec l'œuvre de Vigo, où c'est également sa Juliette qui séduit et est séduite de diverses manières. Tous les personnages masculins du film sont attirés par la belle paysanne. Elle, de son côté, est attiré par son époux Jean, mais, comme Hélène, l'est par Paris / Pâris. Paris la ville qui l'a séduite et va presque la détruire. Hélène et Juliette. Deux femmes désireuses d'aller "au-delà des berges". Deux fantômes qui, inspirés du mythe de l'Atalante, restent toujours pour Nogueira Coelho, des figures de proue dans l'œuvre d'avant-garde de Paulo Emilio et de Jean Vigo.

\section{Le moderne chez Salles Gomes}

Richard Peña, directeur émérite du New York Film Festival, a changé encore une fois de registre, pour nous parler de la modernité dans l'œuvre critique de Salles Gomes. En appliquant son engagement politique à sa carrière de critique du cinéma, Salles Gomes a instauré dans son pays une nouvelle façon de faire de la critique. Il analysait le film non seulement de façon formelle ou par rapport à sa position idéologique (pour ou contre), mais les deux entremêlées, l'art impliquant les questions politiques, historiques ou morales. Pour cela, il s'appuyait sur les théories d'Eisenstein et les critiques de Bazin - deux maîtres représentant bien la tension entre création et enregistrement vécu par lui. Pour lui l'œuvre d'art ne contient pas de signification en soi, et cherche plutôt à dialoguer avec le public. C'est dans ce dialogue que surgit sa véritable signification. Dialogue défendu aussi par Salles Gomes dans sa lutte pour un cinéma moderne qui devrait exiger plus du spectateur. Sa critique d'Umberto $D$ de De Sica est l'illustration parfaite de sa tentative de décrire un cinéma moderne, c'est-à-dire un cinéma de fiction qui permet à la réalité d'être dévoilée au public, tout en dialoguant avec lui. Pour illustrer son hypothèse, il s'appuya sur les articles consacrés aux Quatre cents coups de Truffaut et surtout à Hiroshima mon amour, de Resnais, film qui représentait pour lui un grand pas vers une nouvelle expression cinématographique, grâce à une concentration accrue sur le comportement et la conduite des personnages, un éloignement $\mathrm{du}$ psychologique, sans pour autant chercher à conduire les spectateurs vers des conclusions attendues. Un vrai film moderne.

\section{Paulo Emilio, l'architecte culturel}

Pour conclure ces journées que ponctuaient en outre des projections de films et leurs présentations (l'Atalante, Zéro de conduite - dont Émile Breton analysa la réception critique puis dans les ciné-clubs en 1946 -, Ungaretti em São Paulo de Sérgio Muniz, documentaire de 14 minutes de 1970 où Paulo Emilio présente la ville de São Paulo au poète italien Ungaretti), Sebastiana Gomes Lisboa mit en évidence le travail de Paulo Emilio en tant qu'architecte culturel des relations entre le Brésil et la France, soucieux de favoriser les rencontres entre les cinéphiles de deux pays, ainsi qu'entre tous ceux qui militaient pour la valorisation du cinéma comme objet culturel et document historique. Paulo Emilio a emmené au Brésil tout ce qu'il avait assimilé dans ses séjours en France, où, en compagnie d'un autre Brésilien - le physicien et critique de cinéma Plínio Sissekind Rocha -, il vécut intensément l'action politique à travers les luttes du Front populaire, en même temps que la passion de l'étude du cinéma européen. Le contact avec plusieurs films d'avantgarde soviétique, française et allemande qui n'étaient pas projetés au Brésil à l'époque, ainsi que les rencontres avec des militants politiques comme Victor Serge ou Andrea Caffi lui ont ouvert tout un nouveau champ de possibilités pour ses analyses, approchant encore davantage la poésie et la politique dans sa façon de faire ses critiques. Les films de Jean Vigo ont certainement joué un rôle crucial pour le jeune Brésilien. Lors de ses retours au pays, soit avec la fondation d'un ciné-club au département de philosophie de la USP, soit avec la création de la revue littéraire Clima (au début des années 1940), ou encore avec sa participation à la fondation du premier cours de cinéma à l'Université de Brasília (au début des années 1960), Paulo Emilio a plongé la 
critique cinématographique brésilienne dans le monde de la politique.

Avec un discours qui dénonçait les difficultés de la mise en place d'une cinématographie nationale indépendante dans un pays sous-développé, le critique brésilien a joué un rôle fondamental pour la nouvelle génération de critiques qui apprenaient l'histoire du cinéma à travers ses articles et qui sont devenus les protagonistes du Cinema Novo - ce qui n'est sans doute pas un hasard.

Cela dit, probablement en raison de son appartenance à une génération d'intellectuels engagés dans les ruptures avec les modèles établis, Paulo Emilio prônait plutôt une influence passive d'une culture sur l'autre, avec des échanges et réciprocités culturels, a suggéré Gomes Lisboa. Sans nier l'importance du socialisme et du marxisme pour l'évolution sociale du côté de l'Occident, le critique brésilien défendait l'idée que chaque société avait un bagage culturel important pour sa propre organisation sociale, aucun modèle politique n'étant donc valable universellement. Finalement, dans ces allées et retour entre la France et le Brésil, Paulo Emilio Salles Gomes a joué un rôle de "passeur», c'est-à-dire de responsable du transfert culturel, scientifique et artistique à travers le monde, en faisant le pont entre les cultures, les groupes et les savoirs des uns et des autres, sans jamais vouloir établir une relation de dépendance entre les différentes cultures, mais plutôt de réciprocité. Car, pour lui, dans n'importe quel rencontre il y a forcément des échanges, même dans des situations qui peuvent être représentatives d'une apparente supériorité économique, sociale ou culturelle.

Cette intervention venait ainsi confirmer celle d'Adilson Mendes dans sa "présentation du personnage», au début du colloque, la boucle était donc fermée.
Table ronde avec 1895 revue d'histoire du cinéma: "La restauration des films à l'ère du numérique» (Université Paris 3, 13 mai 2013)

LE 13 MAI DERNIER s'est tenue à l'Université Paris 3 une table ronde avec projection autour de la question de la restauration des films à l'ère du numérique (consultable en ligne sur le site internet de Paris 3 ). Organisée par la Cinémathèque universitaire en partenariat avec l'AFRHC, elle coïncidait avec la sortie du $n^{\circ} 69$ de 1895 revue d'histoire du cinéma qui proposait un dossier "Archives" consacré à ce sujet, ainsi qu'un "Point de vue» de Paolo Cherchi Usai, cofondateur du Giornate del Cinema Muto de Pordenone et conservateur en chef à la George Eastman House. Laurent Véray, président de la Cinémathèque universitaire et membre du conseil d'administration de l'AFRHC, était l'animateur de la soirée et a commencé par exposer les enjeux et les questionnements abordés par les différents intervenants. Le débat portait sur les incidences de l'avènement du numérique, non pas dans le domaine de la production actuelle des images animées, mais plutôt dans ceux de leur conservation et de leur restauration. Selon lui, nous sommes dans une période de transition, et donc d'incertitude, comparable à certains égards, pour les plus alarmistes, à l'arrivée du sonore et du parlant dans les années 1927-1929, qui provoqua la perte d'un savoir-faire ainsi que de nombreux films. Or, la situation semble tout de même assez différente puisqu'il existe aujourd'hui des institutions patrimoniales qui tentent de mener une réflexion sur ces questions posées par l'arrivée du numérique.

Avant la discussion furent projetés le Spectre rouge et Métempsychose (1907) de Segundo de Chomón, le Pêcheur de perles (1907) de Ferdinand Zecca et Segundo de Chomón, et les Hallucinations du baron de Lilia Oliveira Münchhausen (1911) de Georges Méliès, présentés par Laure Gaudenzi, responsable de la Cinémathèque universitaire de Paris 3. Ces quatre films peints au pochoir, projetés ici dans des copies $16 \mathrm{~mm}$ destinées à une diffusion de type ciné-club, étaient impressionnants par la qualité de leurs effets spéciaux et la palette des couleurs utilisées. Une scène du Spectre rouge 